\title{
A CHARACTERIZATION OF QUATERNION PLANES
}

\author{
Dedicated to my teacher, Prof. H. Salzmann, on his 60th birthday
}

ABSTRACT. The eight-dimensional planes admitting $\mathrm{SL}_{2} \mathrm{H}$ as a group of automorphisms are determined.

Every open subset of the projective plane over $\mathbb{R}, \mathbb{C}, \mathbb{H}$ (Hamilton quaternions) or $\mathbb{O}$ (Cayley octonions) carries a stable plane in the sense of [4]. There exist stable planes which cannot be embedded into projective planes: Strambach [11] and Löwen [5] have constructed such planes admitting groups of automorphisms isomorphic to $\mathrm{SL}_{2} \mathbb{R}$ and $\mathrm{SL}_{2} \mathbb{C}$ respectively. For Strambach's plane, there is a generalization beyond the realm of stable planes [7]. Here we show that there is no quaternion analogue of these examples.

THEOREM. Let $\mathbb{M}=(M, \mathscr{M})$ be a locally compact stable plane of topological dimension 8 admitting $\Delta=\mathrm{SL}_{2} \mathbb{H}$ as a group of automorphisms. Then $\mathbb{M}$ contains an open $\Delta$-invariant subplane which is isomorphic to the punctured affine plane over $\mathbb{H}$, and the action of $\Delta$ restricted to this subplane is equivalent to the natural (linear) action on $\mathbb{H}^{2} \backslash\{(0,0)\}$.

REMARKS. (a) The subplane above is the geometry induced on the set of points moved by the central involution $\zeta$ of $\Delta$. Since $\zeta$ cannot be planar, one can show that $\mathbb{M}$ is embedded into the projective plane over $\mathbb{H}$, and that the action of $\Delta$ extends to the natural one.

(b) A special case of the stable planes considered here are compact eightdimensional projective planes. All such planes with automorphism groups of dimension at least 17 have been determined by Salzmann [9]. For semisimple groups, this bound lowers to 16 (see $[9,(1)])$. Our result extends this classification to the case of the 15-dimensional groups locally isomorphic to $\mathrm{SL}_{2} \mathbb{H}$. (Since $\mathrm{SO}_{5} \mathbb{R}$ cannot act on eight-dimensional projective planes, we can exclude $\mathrm{PSL}_{2}$ 田.)

NOTATION. Let $\Delta=\mathrm{SL}_{2} \mathbb{H}$, and let

$$
\Upsilon=\left\{\left(\begin{array}{ll}
1 & 0 \\
c & d
\end{array}\right) \mid d \bar{d}=1\right\} \cong \mathrm{ASL}_{1} \mathbb{H}
$$

be the stabilizer of the point $(1,0)$ in the natural linear action on $\mathbb{H}^{2}$ and 
consider the maximal compact subgroup $\Sigma=\left\{\left(\begin{array}{ll}1 & 0 \\ 0 & d\end{array}\right) \mid d \bar{d}=1\right\}$ of $\Upsilon$. Then

$$
\Upsilon^{*}=\left\{\left(\begin{array}{ll}
1 & b \\
0 & d
\end{array}\right) \mid d \bar{d}=1\right\}
$$

is the stabilizer of the line $\{(1, y) \mid y \in \mathbb{H}\}$. Let

$$
\Phi=\operatorname{SU}_{2} \mathbb{H}=\left\{\left(\begin{array}{ll}
a & b \\
c & d
\end{array}\right) \mid\left(\begin{array}{ll}
a & b \\
c & d
\end{array}\right)\left(\begin{array}{ll}
\bar{a} & \bar{c} \\
\bar{b} & \bar{d}
\end{array}\right)=\left(\begin{array}{ll}
1 & 0 \\
0 & 1
\end{array}\right)\right\} \cong \operatorname{Spin}_{5}
$$

All subgroups to be considered are closed, and all isomorphisms are isomorphisms of topological groups. The line joining two points $x, y$ will be denoted as $x y$.

(1) LEMMA. (a) Every connected subgroup of $\Phi$ not containing the central involution $\zeta=\left(\begin{array}{rr}-1 & 0 \\ 0 & -1\end{array}\right)$ of $\Phi$ is either conjugate to $\Sigma$ or at most onedimensional.

(b) Every subgroup of $\Delta$ isomorphic to $\Upsilon$ is conjugate to $\Upsilon$ or to $\Upsilon^{*}$.

(c) Let $\Lambda$ be a subgroup of $\Delta$ containing $\Sigma$. If the centralizer $\mathrm{C}_{\Lambda}(\alpha)$ of the involution $\alpha=\left(\begin{array}{rr}1 & 0 \\ 0 & -1\end{array}\right) \in \Sigma$ coincides with $\Sigma$, then either the connected component $\Lambda^{1}$ is equal to $\Sigma$, or $\Lambda \cong \Upsilon$.

(d) Any proper subgroup $\Lambda<\Delta$ with $\operatorname{dim} \Delta / \Lambda \leqslant 4$ is conjugate to the group $N=\left\{\left(\begin{array}{ll}a & 0 \\ c & d\end{array}\right) \mid a \bar{a} \bar{d}=1\right\}$. Consequently, the smallest non-trivial orbits of $\Delta$ are four-dimensional, and the action of $\Delta$ on such an orbit is equivalent to the natural action on the projective line $\mathbb{H} \cup\{\infty\} \approx \mathbb{S}_{4}$.

Proof. (a) Let $\Xi$ be a non-trivial connected subgroup of $\Phi$ not containing $\zeta$. There is no pair of commuting involutions in $\Xi$ since their product would be $\zeta$. Therefore $\Xi$ is a compact Lie group of rank 1 , and $\operatorname{dim} \Xi=1$ or $\Xi \cong \operatorname{Spin}_{3}$ (cf. $[1,22 \S 3$, no. 6, Prop. 6]). In the second case, we may assume that $\alpha=\left(\begin{array}{rr}1 & 0 \\ 0 & -1\end{array}\right) \in \Xi$. Then $\Xi$ is a subgroup of the centralizer $C_{\Phi}(\alpha)=$ $\left\{\left(\begin{array}{ll}a & 0 \\ 0 & d\end{array}\right) \mid a \bar{a}=d \bar{d}=1\right\}$. The projections of $\Xi$ to the quasi-simple factors $\Sigma$ and $\Theta=\left\{\left(\begin{array}{ll}a & 0 \\ 0 & 1\end{array}\right) \mid a \bar{a}=1\right\}$ must be bijective or trivial. Since the kernel of the projection to $\Theta$ contains $\alpha$, we have $\Xi=\Sigma$.

(b) Let $\Upsilon \cong \Lambda \leqslant \Delta$ and write $\Lambda=\Xi \Omega$ with $\Xi \cong \operatorname{Spin}_{3}, \Omega \cong \mathbb{R}^{4}$ and 
consider the natural linear action on $\mathbb{G}^{2}=\mathbb{C}^{4}$ as a complex representation. Since $\Omega$ is abelian, there is a vector $v \in \mathbb{C}^{4}$ with $v^{\Omega} \subseteq \mathbb{C} v$ by Lie's theorem. We may assume that $v=(1,0)$. Then $\Omega<\mathrm{N}=\left\{\left(\begin{array}{ll}a & 0 \\ c & d\end{array}\right) \mid a \tilde{a} d \bar{d}=1\right\}$. From $v^{\Omega} \subseteq \mathbb{C} v$ we deduce $\operatorname{dim} \Omega_{v} \geqslant 2$. The stabilizer $\Omega_{v}$ consists of axial collineations of the affine quaternion plane. Since $\Xi$ acts effectively on $\Omega$, all elements of $\Omega$ are axial with axes through the origin. The group $\Delta$ leaves invariant the line at infinity $W$, therefore all centers of elements of $\Omega$ lie on $W$. The homologies in $\Delta$ are contained in compact subgroups. Thus $\Omega$ consists of elations. Commutativity yields $\Omega=\left\{\left(\begin{array}{ll}1 & 0 \\ c & 1\end{array}\right) \mid c \in \mathbb{H}\right\}$. The normalizer of $\Omega$ is $\mathrm{N}$, and we may assume that the compact group $\Xi$ is contained in $\Psi=\left\{\left(\begin{array}{ll}a & 0 \\ 0 & d\end{array}\right) \mid a \bar{a} d \bar{d}=1\right\}$. Acting effectively on $\Omega$, the group $\Xi$ cannot contain $\zeta$. By (a) we have $\Xi=\Sigma$ or $\Xi=\Sigma^{l}$, where $l=\left(\begin{array}{ll}0 & 1 \\ 1 & 0\end{array}\right) \in \Phi$. In the second case, $\Lambda^{\imath}=\Upsilon^{*}$.

(c) Assume that $\Sigma \neq \Lambda^{1}$. By (a) the subgroup $\Sigma$ of $\Lambda^{1}$ is maximal compact. Therefore $\Sigma=\Phi \cap \Lambda^{1}$, and $\operatorname{dim} \Delta / \Lambda=\operatorname{dim} \Delta / \Lambda^{1} \geqslant \operatorname{dim} \Phi / \Sigma=7$. Since $\Delta$ is 15 dimensional, we have $\operatorname{dim} \Lambda \leqslant 8$. Any semisimple linear group with a maximal compact subgroup isomorphic to $\Sigma$ contains a central involution which lies in each maximal compact subgroup (cf. [12]). Thus $\mathrm{C}_{\Lambda}(\alpha)=\Sigma$ implies that $\Lambda$ is not semisimple. Now the Levi decomposition shows that there is a connected solvable group $\Omega$ such that $\Lambda^{1}=\Sigma \Omega$. Let $\mathrm{N}$ be a minimal abelian normal subgroup of $\Lambda^{1}$. Being reductive, the group $\Sigma$ acts completely reducibly on the Lie algebra of $\Omega$. Therefore there is an invariant complement $\mathrm{K}$ of $\mathrm{N}$ in $\Omega$. Since $\Sigma$ acts effectively on $\mathrm{N}$, we have $\mathrm{N} \cong \mathbb{R}^{4}$ and $\operatorname{dim} \mathrm{K} \leqslant 1$. Thus $\Sigma$ acts trivially on $K$, and from the centralizer condition we get $K=\mathbb{1}$. By (b) the connected component $\Lambda^{1}$ is conjugate to $\Upsilon^{*}$ or $\Upsilon^{*}$. The normalizer of $\Upsilon$ in $\Delta$ being the product of $\Upsilon$ and the centralizer $C_{\Delta}(\Sigma)$, we obtain $\Lambda=\Lambda^{1}$.

(d) Let $\mathrm{K}$ be a maximal compact subgroup of the connected component $\Lambda^{1}$. By [6] we have $\operatorname{dim} K=6$. Thus $K$ is locally isomorphic to $\operatorname{Spin}_{4}$ because $\Phi$ has rank 2. Since $\Phi$ does not contain a quadruple of commuting involutions, the group $\mathrm{K}$ is isomorphic to $\mathrm{Spin}_{4}$ and centralizes two involutions. We can assume that $\alpha$ is one of them, and obtain $K=C_{\Phi}(\alpha)$. Now $\Lambda$ has dimension 11. Any semisimple connected linear Lie group containing $\mathrm{Spin}_{4}$ as a maximal compact subgroup centralizes all its involutions (cf. [12]). The Levi decomposition shows that there is a solvable connected invariant subgroup $\Omega$ of $\Lambda$ such that $\Lambda^{1}=\mathrm{K} \Omega$. Considering the usual linear action of $\Delta$ on $\mathbb{H}^{2}=\mathbb{C}^{4}$ as a complex representation, we find $v \in \mathbb{H}^{2}$ such that $v^{\Omega} \subseteq \mathbb{C} v$ (Lie's theorem). If $\Omega$ had two linearly independent eigenvectors, we could 
assume that $\Omega \leqslant\left\{\left(\begin{array}{ll}a & 0 \\ 0 & d\end{array}\right) \mid a \bar{a} d \bar{d}=1\right\}$, a contradiction to the fact that $\Omega$ is homeomorphic to $\mathbb{R}^{5}$ (by the Malcev-Iwasawa decomposition). Normalizing $\Omega$, the group $\Lambda$ is therefore conjugate to a subgroup of $N$. Equality of dimensions yields that $\Lambda=N$ since $N$ is connected.

In order to determine the stabilizer of a line we need the following

(2) LEMMA. Let $\mathbb{M}$ be a locally compact stable plane of finite positive dimension and assume that the lines are (topological) manifolds. Let $\alpha$ be an axial involution and $x$ any point on the axis $A$. Then $\alpha$ fixes precisely one line $Z \neq A$ through $x$.

Proof. We consider the action of $\alpha$ on the line pencil through $x$. By $[3,(1.19)]$ the pencil $\mathscr{M}_{x}$ is homeomorphic to a sphere $S_{l}$. For the restricted action on $\mathscr{M}_{x} \backslash\{A\} \approx \mathbb{R}^{l}$, there is at least one fixed element $Z$ by [10]. Let $X$ be a further fixed line through $x$. Choosing $y \in A$ near $x$, one finds a fixed line $Y$ through $y$ intersecting at least one of the two lines $X, Z$ in a point $z$ outside $A$. Having center $z$, the automorphism $\alpha$ has two axes and is trivial.

PROOF OF THE THEOREM. Without loss of generality we may assume that $M$ is connected and that $\zeta=\left(\begin{array}{rr}-1 & 0 \\ 0 & -1\end{array}\right)$ has no fixed points; in the general case, the fixed points of $\zeta$ form a $\Delta$-invariant closed set which can be removed.

(i) The action of $\Delta$ on $M$ is equivalent to the natural (linear) action of $\Delta$ on $H^{2} \backslash\{0\}$.

Proof. We show that $\Delta_{x} \cong \Upsilon$ for any point $x \in M$. Then all orbits are eightdimensional and thus open. Connectedness yields transitivity, and (1.b) completes the proof of (i).

Since $x \neq x^{5}$, a maximal compact subgroup of the connected component of $\Lambda=\Delta_{x}$ can be assumed to be $\Sigma$ (cf. (1.a)). The centralizer of $\alpha=\left(\begin{array}{rr}1 & 0 \\ 0 & -1\end{array}\right)$ in $\Delta$ is the direct product of the groups

$$
\Sigma, \Theta=\left\{\left(\begin{array}{ll}
a & 0 \\
0 & 1
\end{array}\right) \mid a \bar{a}=1\right\} \text { and } \mathrm{P}=\left\{\left(\begin{array}{cc}
r & 0 \\
0 & r^{-1}
\end{array}\right) \mid r>0\right\} .
$$

Let $G=x x^{\zeta} \in \mathscr{F}_{\zeta}=\left\{y y^{\zeta} \mid y \in M\right\}$. The set $\mathscr{F}_{\zeta}$ is connected and locally homeomorphic to a line (cf. $[4,(1.1)]$ ). Applying (1.d) to the action of $\Phi$ on $\mathscr{F}_{\zeta}$ we obtain transitivity on $\mathscr{F}_{\zeta}$ and see that $\alpha$ fixes precisely two lines $G, H$ of $\mathscr{F}_{\zeta}$. Moreover, the set $\{G, H\}$ is the set of fixed lines of $\Sigma \Theta$ in $\mathscr{F}_{\zeta}$. 
Next, we determine the geometric type of $\alpha$. If $\alpha$ is a central involution, its center has to be fixed by $\zeta$. Analogously, no line $L \neq G$ through $x$ can be an axis of $\alpha$. Planarity of $\alpha$ would yield a two-dimensional set of fixed lines in $\mathscr{F}_{\zeta}$. In the only remaining case, the involution $\alpha$ has axis $G$ and acts freely on $M \backslash G$. Via conjugation by $l=\left(\begin{array}{ll}0 & 1 \\ 1 & 0\end{array}\right) \in \Phi$ we obtain the same result for $-\alpha$ and $H$.

For any point $y \in G$ we have $\Phi_{y} \leqslant \Phi_{G}$, and the connected component of $\Phi_{y}$ is $\Sigma$ or $\Theta$ by (1.a). Since $-\alpha$ acts freely on $M \backslash H$, we obtain that $\Sigma$ is contained in the group $\Phi_{[G]}=\left\{\varphi \in \Phi_{G} \mid \varphi\right.$ acts trivially on $\left.G\right\}$. Again, conjugation by $\imath$ shows that $\Theta \leqslant \Phi_{[H]}$. Free action outside the axes enforces equality in both cases.

For any point $y \in G$ we consider the stabilizer in $\Sigma \Theta P=\mathrm{C}_{\Delta}(\alpha)$. Since $l$ induces inversion on $\mathrm{P}$, we have $\mathrm{P}_{y}=\mathrm{P}_{y}^{-1}=\mathrm{P}_{y}=\mathrm{P}_{y^{z}}$. The stabilizer $\mathrm{P}_{y}$ therefore acts trivially on the three-dimensional orbits $y^{\Theta}$ and $y^{i \Sigma}$. Since these two orbits generate the whole plane, one gets $P_{y}=1$. Now $(\Sigma \Theta P)_{x}=\Sigma(\Theta P)_{x}$. The kernel of the projection of $(\Theta P)_{x}$ to $\Theta$ lies in $\mathrm{P}_{x}=1$, therefore $(\Theta \mathrm{P})_{x} \leqslant$ $\Theta_{x}=1$. Finally, (1.c) shows that $\Delta_{x} \cong \Upsilon$.

(ii) Let $x \in M$ and $\Delta_{x}=\Upsilon=\Sigma \Omega$ with $\Omega=\left\{\left(\begin{array}{ll}1 & 0 \\ c & 1\end{array}\right) \mid c \in \mathbb{H}\right\}$. Then $\Omega$ is sharply transitive on $\mathscr{M}_{x} \backslash\left\{x x^{\zeta}\right\}$.

Proof. Let $L \in \mathscr{M}_{x} \backslash\left\{x x^{\zeta}\right\}$. In a positive-dimensional stabilizer $\Omega_{L}$ one finds a one-parameter group $\Xi=\left\{\left(\begin{array}{cc}1 & 0 \\ r c & 1\end{array}\right) \mid r \in \mathbb{R}\right\}$. This group is invariant under $\mathrm{P}=\left\{\left(\begin{array}{cc}r & 0 \\ 0 & r^{-1}\end{array}\right) \mid r>0\right\}$ and therefore acts trivially on the orbit $L^{\Omega \mathrm{P}}$. If $L^{\Omega}$ is not trivial, there are $\rho \in \mathrm{P}$ and $\omega \in \Omega$ such that $L^{\rho}$ and $L^{\omega}$ meet outside $x x^{\zeta}=G$. This contradicts the fact that $\Omega$ acts freely outside $G$. Thus $\Omega=\Omega_{L}$. For any further line $L^{\prime}$ fixed by $\Omega$ there is again $\rho \in \mathrm{P}$ such that $L^{\prime}$ and $L^{\rho}$ meet outside $G$. Since all four-dimensional orbits in $\mathscr{M}_{x}$ are open, this yields a (sharply) transitive action of $\Omega \cong \mathbb{R}^{4}$ on $\mathscr{M}_{x} \backslash\{G, L\}$, which contradicts $\mathscr{M}_{x} \approx S_{4}$.

(iii) The stabilizer of any line $L \neq L^{\zeta}$ is isomorphic to $\Upsilon$.

Proof. By the preceding results, one can assume that $L=Z$ (compare (2)). Thus $\Sigma=\Delta_{x, L}$. By (1.a) $\Sigma$ is a maximal compact subgroup of $\Delta_{L}^{1}$. For any $\rho \in \Delta_{L}$ commuting with $\alpha=\left(\begin{array}{rr}1 & 0 \\ 0 & -1\end{array}\right) \in \Sigma$ we have $x^{\rho}=x^{\rho \alpha}$. Since $\alpha$ acts freely outside $x x^{\zeta}$, we obtain $\rho \in \Delta_{x, L}=\Sigma$ and $\Delta_{L} \cong \Upsilon$ by (1.c).

Since $\Delta_{x, L}=\Sigma \neq \Delta_{L}$, we have $\Delta_{x} \neq \Delta_{L}$. The maximal compact subgroup $\Sigma$ of 
$\Delta_{L}$ is also maximal compact in $\Upsilon$ and in $\Upsilon^{*}$. Therefore there is $\delta \in N_{\Delta}(\Sigma)$ such that $\Delta_{L}=\Upsilon^{\delta}$ or $\Delta_{L}=\Upsilon^{* \delta}$. But the normalizer of $\Sigma$ in $\Delta$ is precisely the centralizer of $\alpha$, which leaves both $\Upsilon$ and $\Upsilon^{*}$ invariant. Therefore $\Delta_{L}=\Upsilon^{*}$. Transitivity of $\Delta$ on points and lines moved by $\zeta$ allows the reconstruction of the geometry analogously to [2]. This concludes the proof.

\section{REFERENCES}

1. Bourbaki, N., Groupes et algèbres de Lie, Chap. IX, Masson, Paris, 1982.

2. Higman, D. G. and McLaughlin, J. E., 'Geometric ABA-groups', Illinois J. Math. 5 (1961), $382-397$.

3. Löwen, R., 'Vierdimensionale stabile Ebenen', Geom. Dedicata 5 (1976), 239-294.

4. Löwen, R., 'Halbeinfache Automorphismengruppen von vierdimensionalen stabilen Ebenen sind quasi-einfach', Math. Ann. 236 (1978), 15-28.

5. 'Löwen, R., 'Actions of Spin $_{3}$ on 4-dimensional stable planes', Geom. Dedicata 21 (1986), 1-12.

6. Mann, L. N., 'Gaps in the dimensions of transformation groups', Illinois J. Math. 10 (1966), $532-546$.

7. Prażmowski, K., 'An axiomatic description of the Strambach planes', Geom. Dedicata 32 (1989), 125-156.

8. Richardson, R. W., 'Groups acting on the 4-sphere', Illinois J. Math. 5 (1961), 474-485.

9. Salzmann, H., 'Compact 8-dimensional projective planes', Forum Math. 2 (1990), 15-34.

10. Smith, P. A., 'Fixed-point theorems for periodic transformations', Amer. J. Math. 63 (1941), $1-8$.

11. Strambach, K., 'Zur Klassifikation von Salzmann-Ebenen mit dreidimensionaler Kollineationsgruppe', Math. Ann. 179 (1968), 15-30.

12. Tits, J., 'Tabellen zu den einfachen Lie Gruppen und ihren Darstellungen', Lecture Notes in Math. 31, Springer, New York, 1967.

Author's address:

Markus Stroppel,

Mathematisches Institut der Universität Tübingen, Auf der Morgenstelle 10,

D-7400 Tübingen 1,

F.R.G.

(Received, January 19, 1990) 